- Desalination and Water Treatment

227 (2021) 163-176

www.deswater.com

July

(O) doi: $10.5004 /$ dwt.2021.27307

\title{
Electrocoagulation-flotation treatment followed by sedimentation of carpet cleaning wastewater: optimization of key operating parameters via RSM-CCD
}

\author{
Elena Shakeria,b,t, Milad Mousazadeh ${ }^{\mathrm{a}, b, t}$, Hedieh Ahmadparic ${ }^{c}$, Işık Kabdaşli ${ }^{\mathrm{d}}$, Hamzeh Ali \\ Jamalie, Nuno S.Graça ${ }^{\mathrm{f}}$, Mohammad Mahdi Emamjomehe,* \\ eStudent Research Committee Qazvin University of Medicnl Sciences, Qazvin, Iran, emails: shakeri.elenaeyahoo.com (E. Shakeri), \\ m.milad199393egmail.com (M. Mousazadeh) \\ 'Department of Environmental Health Engineering, School of Health Quzvin Lniversity of Medical Sciences, Quzvin. Iran \\ Department of Irrigation and Drainage, College of Aburaihan, University of Tehran. Iran, email: h.ahmadpariegmail.com \\ ¿İstanbul Technical University, Civil Engineering Faculty. Environmental Engineering Department, Aynzağa Campus, 34469 Maslak. \\ İstanbul, Republic of Turkey, email: kabdaslieitu.edu.tr \\ "Social Detenninants of Health Research Center, Research Institute for Prevention of Non-Communicable Diseases, Qazvin Lniversity \\ of Medical Sciences, Qazvin, Iran, emails: jomalisadraeiegmail.com (H. Ali Jamali), m_emanjomeheyahoo.com (M.M. Emamjomeh) \\ Laboratory of Separation axd Reaction Engineering - Laboratory of Catalysis and Materials (LSRE-LCM), Department of Chemical \\ Engineering, Lniversity of Porto, Rua Dr. Roberto Frias, s/n, 4200-465 Porto, Portugal, email: nunopsg@efe.up.pt
}

Received 13 January 2021; Accepted 8 April 2021

A B STRACT

In the present study, the treatment of carpet cleaning wastewater was optimized for electrocoagulation-flotation (ECF) followed sedimentation. In the experimental study, an ECF reactor equipped with four monopolar, parallel-connected aluminum electrodes was utilized. For the optimization, the process variables were selected as methylene blue active substance (MBAS), chemical oxygen demand (COD), and turbidity removal efficiencies, along with the characterization of sludge settling volume at $60 \mathrm{~min}$ (SSV). For this goal, response surface methodology (RSM) under central composite design (CCD) was employed to optimize the critical factors viz. $\mathrm{pH}(3.64-10.36)$, current intensity (0.66-2.34 A), and electrolysis time ( $9.55-110.45$ min). RSM-CCD optimized these key factors to achieve maximum removal efficiencies and minimize SSV ${ }_{S a}$. Based on the RSM-CCD prediction, the optimum operating conditions were as $\mathrm{pH}$ of 5.1 , the current intensity of $2 \mathrm{~A}$, and electrolysis time of $53.5 \mathrm{~min}$, in which the obtained model predicted $83.56 \%, 82.54 \%, 88.14 \%$, and $226.22 \mathrm{~mL} / \mathrm{L}$ for MBAS, COD, turbidity, and $\mathrm{SSV}_{e \mathrm{~T}}$ Correspondingly, the predictions were in agreement with the actual results ( $85.50 \%, 84.35 \%, 90.50 \%$, and $240.17 \mathrm{~mL} / \mathrm{L}$, respectively). The operating cost in the optimal conditions was calculated as $0.673 \mathrm{USD} / \mathrm{m}^{3}$. The results of the study indicated that the electrocoagulation-flotation followed sedimentation was a cost-effective treatment process in removing target pollutants from the carpet cleaning wastewater.

Keywords: Electrocoagulation-flotation; Carpet cleaning wastewater; Aluminum electrodes; Optimization; Sludge settling volume

\section{Introduction}

Every year in the world, the carpet cleaning industry, for its various processes, uses a large amount of water According to a study conducted in Iran, about $30 \mathrm{~L}$ of

* Corresponding author.

'These authors contributed equally to this work. water per square meter is used in various carpet cleaning [1]. Wastewater from washing industries such as laundry, carpet cleaning, and car wash offers combinations of different levels, including suspended solids, turbidity, chemical oxygen demand (COD), and surfactants. Surfactants,

1944-3994/1944-3986 (e) 2021 Desalination Publications. All rights reserved. 